\title{
Neutralization of vascular endothelial growth factor antiangiogenic isoforms or administration of proangiogenic isoforms stimulates vascular development in the rat testis
}

\author{
Michelle M Baltes-Breitwisch, Robin A Artac, Rebecca C Bott, Renee M McFee, \\ Jill G Kerl, Debra T Clopton and Andrea S Cupp \\ Department of Animal Science, University of Nebraska-Lincoln, 3934 Fair Street, Lincoln, \\ Nebraska 68583-0908, USA \\ Correspondence should be addressed to A S Cupp; Email: acupp2@unl.edu
}

\begin{abstract}
Vascular endothelial growth factor A (VEGFA) plays a role in both angiogenesis and seminiferous cord formation, and alternative splicing of the Vegfa gene produces both proangiogenic isoforms and antiangiogenic isoforms (B-isoforms). The objectives of this study were to evaluate the expression of pro- and antiangiogenic isoforms during testis development and to determine the role of VEGFA isoforms in testis morphogenesis. Quantitative RT-PCR determined that Vegfa_165b mRNA was most abundant between embryonic days 13.5 and 16 (E13.5 and 16; $P<0.05)$. Compared with ovarian mRNA levels, Vegfa_120 was more abundant at E13-14 $(P<0.05)$, Vegfa_164 was less abundant at E13 $(P<0.05)$, and Vegfa_165b tended to be less abundant at E13 $(P<0.09)$ in testes. Immunohistochemical staining localized antiangiogenic isoforms to subsets of germ cells at E14-16, and western blot analysis revealed similar protein levels for VEGFA_165B, VEGFA_189B, and VEGFA_206B at this time point. Treatment of E13 organ culture testes with VEGFA_120, VEGFA_164, and an antibody to antiangiogenic isoforms (anti-VEGFAxxxB) resulted in less organized and defined seminiferous cords compared with paired controls. In addition, $50 \mathrm{ng} / \mathrm{ml}$ VEGFA_120 and VEGFA_164 treatments increased vascular density in cultured testes by 60 and $48 \%$ respectively, and treatment with VEGFAxxxB antibody increased vascular density by $76 \%$ in testes $(0.5 \mathrm{ng} / \mathrm{ml})$ and $81 \%$ in ovaries $(5 \mathrm{ng} / \mathrm{ml})$ compared with controls $(\boldsymbol{P}<\mathbf{0 . 0 5})$. In conclusion, both pro- and antiangiogenic VEGFA isoforms are involved in the development of vasculature and seminiferous cords in rat testes, and differential expression of these isoforms may be important for normal gonadal development. Reproduction (2010) 140 319-329
\end{abstract}

\section{Introduction}

Two hallmarks of testis morphogenesis are seminiferous cord formation and testis-specific vascular development. The first step in seminiferous cord formation is the expression of Sry from the Sertoli cell which occurs between embryonic days 10.5 and 12.5 (E10.5 and 12.5) in the mouse (Hacker et al. 1995). Expression of Sry promotes the expression of Sertoli cell-specific genes, such as Sox9 (Kidokoro et al. 2004), which then stimulate the proliferation of Sertoli cells and aggregation of Sertoli and primordial germ cells (McLaren 1991, Capel et al. 1999). The next step in seminiferous cord formation involves the migration of cells from the mesonephros into the developing testis and the resulting formation of cords and testis-specific vasculature. These events occur around E11.5-12.5 in the mouse (Tilmann \& Capel 1999, Brennan et al. 2002). Distinct, malespecific vasculature which includes the large coelomic vessel develops concurrently with seminiferous cord formation, and is evident in the mouse testis by E12.5 (Brennan et al. 2002). In the rat, seminiferous cords begin to develop at E13.5 (Magre \& Jost 1980, Jost et al. 1981), a continuous basal lamina which surrounds and delineates cords first appears at E14.5 (Magre \& Jost 1991), and this basal lamina is found throughout the rat testis by E15.5 (Magre \& Jost 1991).

Sertoli cells within the developing testis are thought to drive the migration of mesonephric cells by producing paracrine growth factors which act as chemoattractants (Martineau et al. 1997). Culturing E11.5 mouse gonads separate from mesonephroi impairs cord development (Buehr et al. 1993), suggesting that mesonephric cell migration is crucial to proper cord formation. It has been proposed that these migrating cells are endothelial and pre-peritubular cells (Merchant-Larios et al. 1993); however, other studies have suggested that only cells which are positive for endothelial cell markers migrate from the mesonephros into the $\mathrm{XY}$ gonad (Cool et al. 2008, Combes et al. 2009). Previous studies in our laboratory have demonstrated that vascular endothelial growth factor $\mathrm{A}$ (VEGFA) plays a role in both angiogenesis and seminiferous cord formation in the developing testis. 
Treatment with VEGFA antagonists or VEGFA receptor signal transduction inhibitors reduced vascular density and perturbed, or totally inhibited, cord formation in E13 rat organ culture testes (Bott et al. 2006).

The Vegfa gene consists of eight exons separated by seven introns. Alternative splicing of the Vegfa gene produces different mRNA splice variants and, thus, different protein isoforms with varying numbers of amino acids. Rodent VEGFA isoforms have one less amino acid per isoform than human VEGFA, and each isoform has unique functions depending on its composition and diffusion properties (Park et al. 1993, Ferrara et al. 2003). Differences in diffusion allow VEGFA isoforms to form a chemoattractant gradient, promoting the migration of endothelial cells for neovascularization in developing organs or tumors (Grunstein et al. 2000, Springer et al. 2000).

All VEGFA isoforms were once thought to be proangiogenic; however, antiangiogenic isoforms have now been identified. The antiangiogenic isoform, VEGFA_165B, was first discovered in human renal cells (Bates et al. 2002), and it has been proposed that every proangiogenic isoform has a corresponding antiangiogenic isoform where exon 8a is substituted by 8b (Woolard et al. 2004). Our laboratory recently identified and sequenced mRNA for antiangiogenic isoforms (Vegfa_165b and Vegfa_189b) in rat ovaries, and through these sequences, it determined that these rat antiangiogenic isoforms (VEGFA_165B and VEGFA_189B) have an additional amino acid residue compared with their respective proangiogenic isoforms (VEGFA_164 and VEGFA_188; Artac et al. 2009).

It has been shown that human VEGFA_165B binds with the same affinity as VEGFA_165 to kinase insert domain protein receptor (KDR, also known as VEGFR2 and FLK1) but does not allow for subsequent activation of signaling pathways. Additionally, VEGFA_165B inhibits VEGFA_165-mediated angiogenesis, and VEGFA_165Bexpressing tumors grow more slowly than VEGFA_165expressing tumors (Woolard et al. 2004). Therefore, it appears that the antiangiogenic VEGFA isoforms may inhibit the actions of the proangiogenic isoforms by blocking their binding to the VEGFA receptors, KDR and FMS-like tyrosine kinase 1 (FLT1, also known as VEGFR1).

We have previously localized VEGFA expression to Sertoli and germ cells, and demonstrated Vegfa_120, Vegfa_164, and Vegfa_188 mRNA expression in developing testes (Bott et al. 2006). The first objective of the current study was to identify the expression of the antiangiogenic isoforms and to further evaluate the expression patterns of the proangiogenic isoforms during testis development. The second objective of this study was to investigate the role of VEGFA isoforms in testis morphogenesis. Specifically, we sought to compare the effects of treatment with proangiogenic VEGFA isoforms and neutralization of antiangiogenic VEGFA isoforms on vascular development and cord formation.

\section{Results}

\section{Vegfa isoform mRNA expression during testis development}

Conventional RT-PCR was used to evaluate Vegfa antiangiogenic isoform mRNA expression in developing rat testes. Five developmental time points were evaluated (E13, E14, E16, E18, and postnatal day 0 (P0)). There was no detectable expression of Vegfa_165b prior to cord formation (E13) but Vegfa_165b was present after cord formation at E14, E16, E18, and P0.

Quantitative RT-PCR (QRT-PCR) was performed on seven developmental time points (E13, E13.5, E14, E16, $\mathrm{E} 18, \mathrm{P} 0$, and $\mathrm{P} 3$ ) during testis development to determine mRNA abundance for Vegfa_120, Vegfa_164, and Vegfa_165b. Levels for Vegfa_120 mRNA decreased from E13 to E16 $(P<0.02)$, increased from E16 to E18 $(P<0.04)$, decreased from E18 to P0 $(P<0.04)$, and then increased from P0 to P3 $(P<0.0001$; Fig. $1 \mathrm{~A})$. mRNA levels for Vegfa_164 increased from E13 to E13.5-14 $(P<0.05)$, increased from E14 to E16 $(P<0.003)$, decreased from E16 to E18-P0 $(P<0.002)$, and then decreased again from E18-P0 to P3 ( $P<0.03$; Fig. $1 \mathrm{~B})$. Levels for Vegfa_165b mRNA were greater at E13.5, E14, and E16 compared with all other time points analyzed (Fig. 1C; $P<0.05$ ).

We then compared mRNA levels for Vegfa_120, Vegfa_164, and Vegfa_165b between testes and ovaries at E13 and E14 to pinpoint any differences in Vegfa isoforms at the developmental time point when endothelial cells are migrating from the mesonephros to establish vasculature, and seminiferous cords are forming in the developing testis. No cell migration occurs in the ovary at these time points. The ovarian data used for this comparison were taken from previously published QRT-PCR studies from our laboratory (Artac et al. 2009, McFee et al. 2009). The same primers, probes, and conditions were used for each isoform in both the testis and ovarian experiments. mRNA levels for Vegfa_120 were significantly lower in ovaries than in testes at both E13 $(P<0.0001)$ and E14 $(P<0.03$; Fig. 2A). At E13, Vegfa_164 mRNA levels were greater in ovaries than in testes $(P<0.05)$; however, there was no difference in levels at E14 (Fig. 2B). Levels for Vegfa_ $165 \mathrm{~b}$ mRNA tended to be greater in ovaries than in testes at E13 $(P<0.09)$ but there was no difference between testes and ovaries at E14 (Fig. 2C).

\section{Western blot analysis of VEGFA isoforms during testis development}

Western blot analysis was used to evaluate the presence of VEGFA isoforms in developing rat testes. Five developmental time points were evaluated: E13, E16, E18, P0, and P4 (Fig. 3A). The primary antibody that was used for these western blots will detect all VEGFA isoforms, including antiangiogenic 


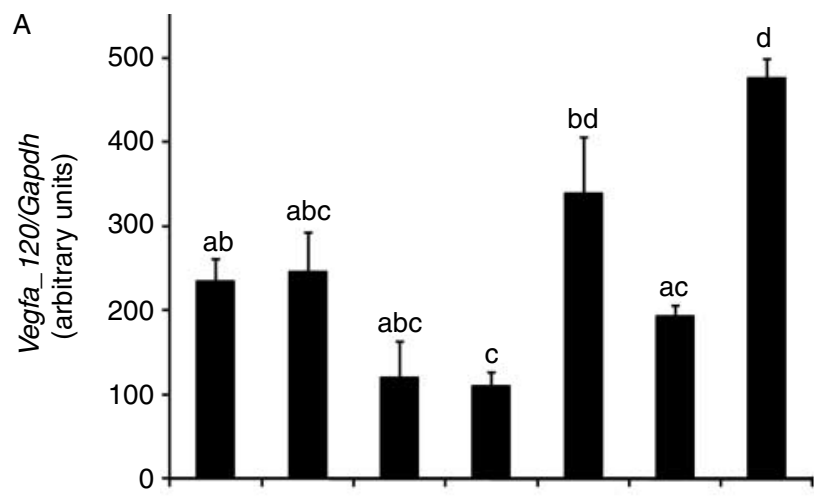

B

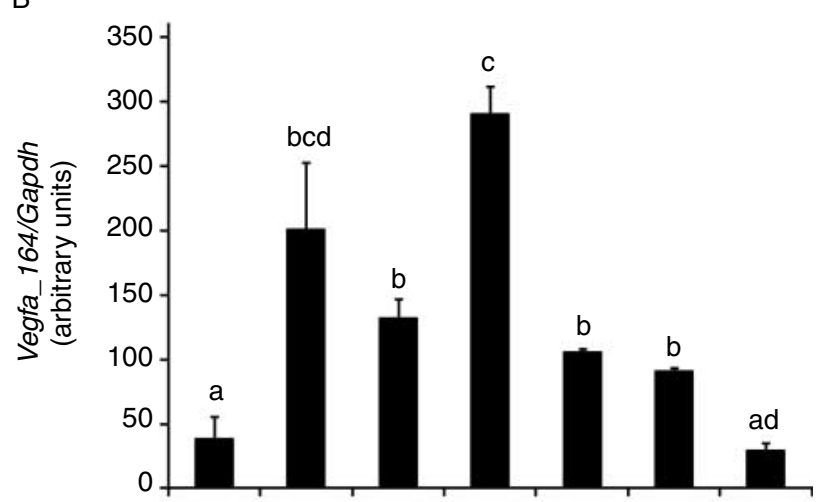

C

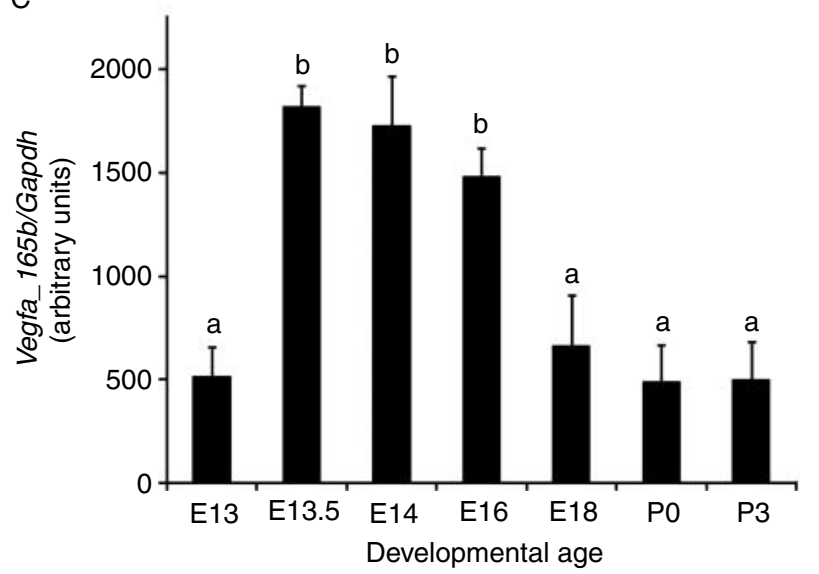

Figure 1 Quantitative RT-PCR for Vegfa_120 (A), Vegfa_164 (B), and Vegfa_165b (C) from E13 through P3 of testis development. Gapdh was used as an endogenous control to account for differences in starting material. These data are the result of at least three different pools of each age tissue. The mean \pm s.E.M. normalized QRT-PCR values are presented for each developmental age. Developmental ages are labeled with letters to represent statistical comparisons: ages labeled with a common letter are not different while ages without a common letter are significantly different $(P<0.05)$.

isoforms. Consequently, the bands detected for VEGFA_120, VEGFA_164, VEGFA_188, and VEGFA_ 205 may also contain the similarly sized antiangiogenic isoforms: VEGFA_121B, VEGFA_165B, VEGFA_189B, and VEGFA_206B respectively. Quantitative analysis demonstrated that VEGFA_120/121B was greater in abundance than VEGFA_164/165B, VEGFA_188/189B, and VEGFA_205/206B at E18 ( $P<0.02$; data not shown). The abundance of VEGFA_205/206B was greater at P4 than that at E13-E16 $(P<0.05$; Fig. 3E). Additionally, the values for VEGFA_188/189B tended to be greater at P4 than the values at E13-E16 $(P<0.09$; Fig. 3D). No statistical differences were detected among isoforms at E13, E16, P0, and P4 or in the abundance of VEGFA_120/121B (Fig. 3B) and VEGFA_164/165B (Fig. 3C) across developmental ages.

A second western blot was performed using an antibody made to the region of exon $8 \mathrm{~b}$ to evaluate the presence of only the VEGFA antiangiogenic isoforms in E13-P4 rat testes (Fig. 4A). Protein bands consistent with VEGFA_121B, VEGFA_165B, VEGFA_189B, and VEGFA_206B were present. Although Vegfa_165b and Vegfa_189b have been previously recognized in rats via RT-PCR (Artac et al. 2009) and VEGFA_121B has been isolated in human tissues (Rennel et al. 2009), this is the first time that the antiangiogenic isoform, VEGFA_206B,
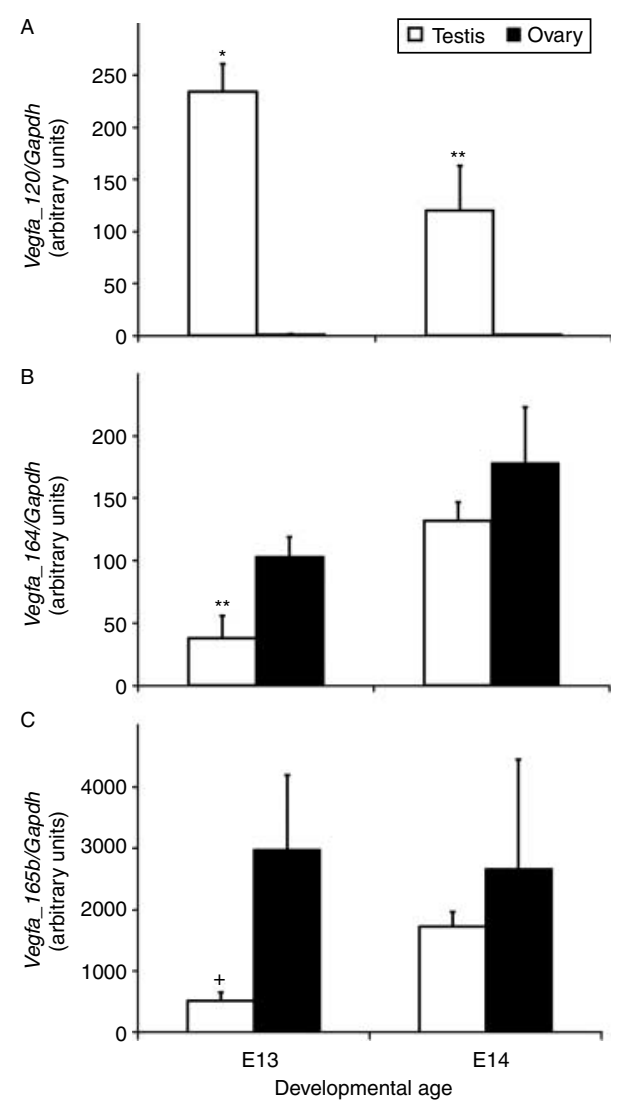

Figure 2 Comparison of quantitative RT-PCR values between E13 and E14 testes and ovaries for Vegfa_120 (A), Vegfa_164 (B), and Vegfa_165b (C). Gapdh was used as an endogenous control to account for differences in starting material. These data are the result of at least three different pools of each age tissue. The mean \pm S.E.M. normalized QRT-PCR values are presented for each developmental age. Asterisks represent a statistically significant difference between testes and ovaries at each age $\left({ }^{*} P<0.0001,{ }^{* *} P<0.05\right)$. The plus sign indicates a tendency toward different means $\left({ }^{+} P<0.09\right)$. 
A
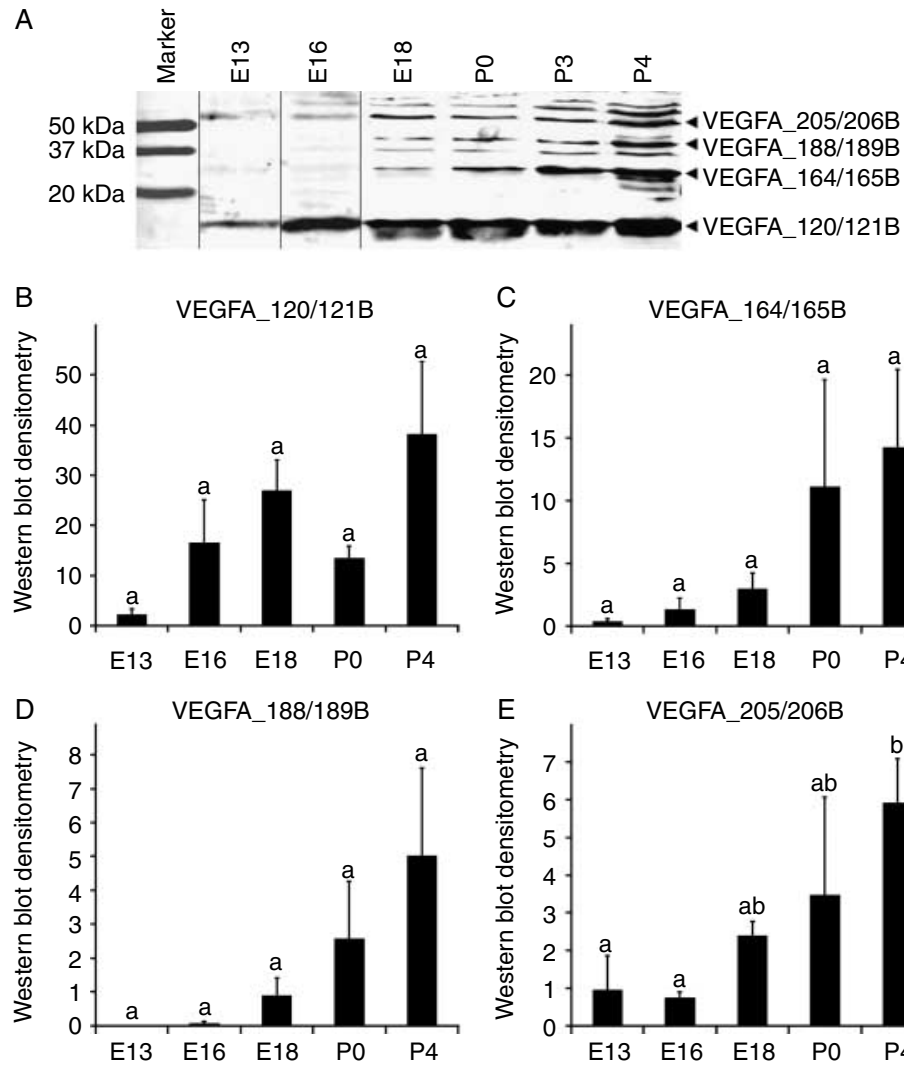

C
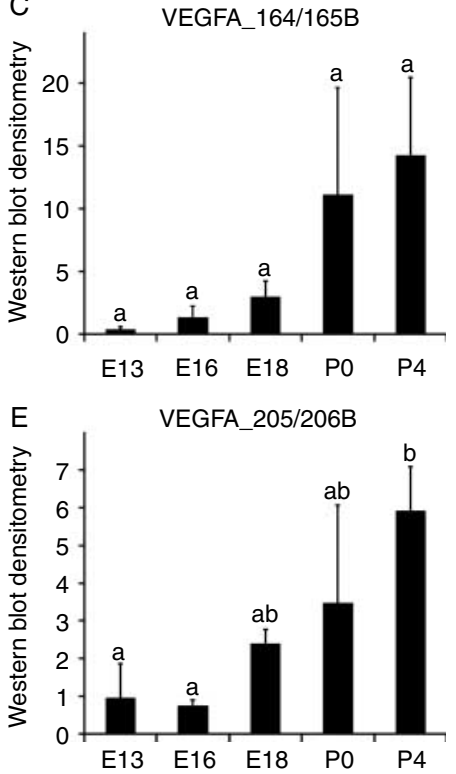

Figure 3 (A) Western blot analysis for VEGFA isoforms from E13 to P4 of testis development. Black lines indicate where different blot images have been spliced together. Recombinant VEGFA_164 served as a positive control (data not shown). Quantitative analysis of western blot bands for VEGFA_120/ 121B (B), VEGFA_164/165B (C), VEGFA_188/189B (D), and VEGFA_205/206B (E) based upon a standard curve created from recombinant VEGFA_164. These data are the result of at least three different pools of each age tissue. The mean \pm s.E.M. densitometry values are presented for each developmental age. Developmental ages are labeled with letters to represent statistical comparisons: ages labeled with a common letter are not different while ages without a common letter are significantly different $(P<0.05)$. has been identified. Quantification of bands revealed that VEGFA_165B was more abundant at P0 than VEGFA_189B $(P<0.04$; data not shown). No statistical differences were detected among isoforms at any of the other developmental time points evaluated (data not shown). The abundance of VEGFA_189B was greater at P4 than the abundance at E16-18 $(P<0.04$; Fig. 4D). There were no statistical differences in the abundance of VEGFA_121B (Fig. 4B), VEGFA_165B (Fig. 4C), and VEGFA_206B (Fig. 4E) across all ages evaluated, likely due to the high variation in protein levels across individual samples.

\section{Localization of VEGFA antiangiogenic isoforms within developing testes}

Immunohistochemistry was performed on E14, E16, and $\mathrm{P} 0$ rat testes to localize the expression of antiangiogenic VEGFA isoforms to specific cell types and tissue locations (Fig. 5). At E14 (Fig. 5B) and E16 (Fig. 5C), the expression of antiangiogenic isoforms was detected in some, but not all, germ cells. Positive staining was also present within the mesonephros (Fig. 5C). In P0 testes, high intensity staining was present within the interstitium with faint, or no staining was visible in Sertoli and germ cells (Fig. 5D). No staining was identified in testis sections that were processed without primary antibody (Fig. 5A).

\section{Effects of recombinant VEGFA_120 and VEGFA_164 on rat testis organ cultures}

Treatment of E13 testis organ cultures with $50 \mathrm{ng} / \mathrm{ml}$ of recombinant VEGFA_120 (Fig. 6D) or VEGFA_164 (Fig. 6G) perturbed seminiferous cord formation in the majority of treated testes based on visual evaluation of brightfield images of whole cultured organs. The cords in treated testes were undefined and poorly organized compared with the cords in control testes (Fig. 6A). Neither the depth (determined by the total number of Z-series confocal microscopy images taken of each organ) nor the area of testes treated with VEGFA_120 or VEGFA_164 was significantly different from control testes (data not shown). The vascular density within VEGFA_120 (Fig. 6E and F) and VEGFA_164 (Fig. 6H and I) treated testes was $60 \%$ (Fig. $7 \mathrm{~A} ; P<0.03$ ) and $48 \%$ (Fig. 7A; $P<0.05)$ greater respectively compared with controls (Fig. $6 \mathrm{~B}$ and $\mathrm{C}$ ).

\section{Effects of VEGFAxxxB antibody on rat testis organ cultures}

The seminiferous cords in the majority of testes treated with $0.5 \mathrm{ng} / \mathrm{ml}$ (Fig. 6J) and $5 \mathrm{ng} / \mathrm{ml}$ (Fig. 6M) of VEGFAxxxB antibody were also less defined and organized than the cords in control testes (Fig. 6A). There was no difference in depth or area between testes 


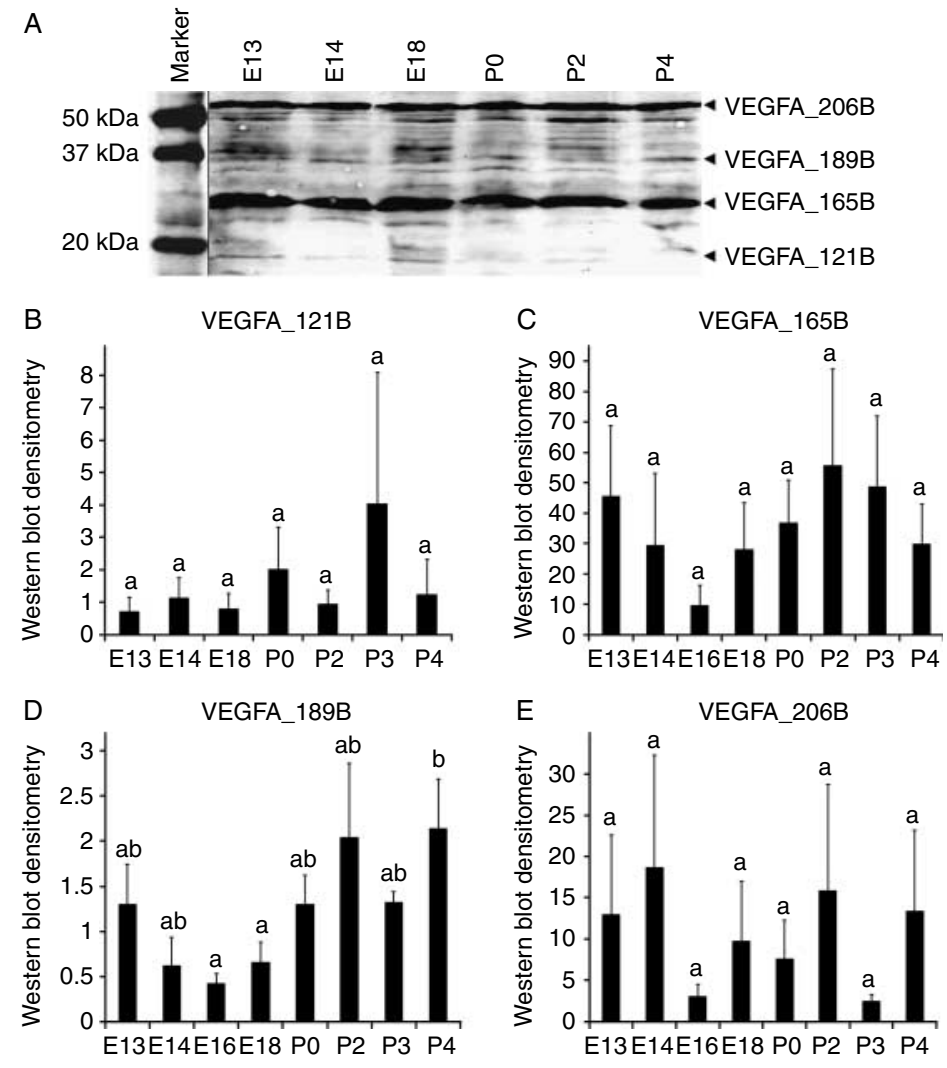

Figure 4 (A) Western blot analysis for antiangiogenic VEGFA isoforms from E13 to P4 of testis development. Recombinant VEGFA_165B served as a positive control (data not shown). Quantitative analysis of western blot bands for VEGFA_121B (B), VEGFA_165B (C), VEGFA_189B (D), and VEGFA_206B (E) based upon a standard curve created from recombinant VEGFA_165B. These data are the result of at least three different pools of each age tissue. The mean \pm S.E.M. densitometry values are presented for each developmental age. Developmental ages are labeled with letters to represent statistical comparisons: ages labeled with a common letter are not different while ages without a common letter are significantly different $(P<0.05)$. treated with either dose of the VEGFAxxxB antibody and testes treated with nonspecific IgG (data not shown). The vascular density of testes treated with $0.5 \mathrm{ng} / \mathrm{ml}$ of VEGFAxxxB antibody (Fig. 6K and L) was increased by $76 \%$ (Fig. $7 ; P<0.008$ ) compared with controls (Fig. 6B and $\mathrm{C}$ ). The vascular density of testes treated with the $5 \mathrm{ng} / \mathrm{ml}$ dose of VEGFAxxxB antibody (Fig. $6 \mathrm{~N}$ and O) tended to be different than controls (Fig. 6B and C) but was not significantly different due to a high variation among samples (Fig. 7; $P<0.09$ ).

\section{Discussion}

The current study is the first to report a role for both proangiogenic and antiangiogenic VEGFA isoforms in the regulation of vasculature and seminiferous cords in the developing rat testis. Neutralization of antiangiogenic isoforms through the use of a VEGFAxxxB antibody resulted in an increase in vascular density, suggesting that removal of antiangiogenic VEGFA isoforms is a powerful method to regulate vascular development. Furthermore, an imbalance in the production of VEGFA pro- versus antiangiogenic isoforms may severely impair testis morphology and development.

Our laboratory has previously demonstrated the presence of Vegfa proangiogenic isoform mRNA expression during testis development (Bott et al. 2006), and in this study, we quantified Vegfa_120 and
Vegfa_164 mRNA across the same developmental ages. mRNA levels for Vegfa_120 in the testis decreased from E13-13.5 to E14-16, while VEGFA_120 protein was the most abundant isoform in embryonic testes after E13. The VEGFA_120 isoform is highly diffusible and is believed to be primarily involved in the recruitment of endothelial cells (Grunstein et al. 2000). During testis development, endothelial cells, resulting from the breakdown of mesonephric vasculature, migrate into the testis and reaggregate to form the coelomic vessel. During this process, these endothelial cells only migrate between the regions where cords are forming, further establishing testis-specific vascular patterns (Coveney et al. 2008). The decrease in abundance of Vegfa_120 mRNA after E13-13.5 is likely due to a reduced requirement for VEGFA_120 after mesonephric endothelial cell migration.

mRNA abundance for Vegfa_164 was greatest at E16 in the testis. The VEGFA_164 isoform is responsible for recruiting endothelial cells and promoting the formation of major blood vessels (Grunstein et al. 2000, Ferrara et al. 2003). The developing XY gonad recruits endothelial cells from the adjacent mesonephros. These migrating endothelial cells contribute to the formation of the arterial system within the testis, establishing a new pattern of blood flow (Brennan et al. 2002). It has been demonstrated that the proliferation of Sertoli cells increases dramatically after E14, and there is extensive proliferation 

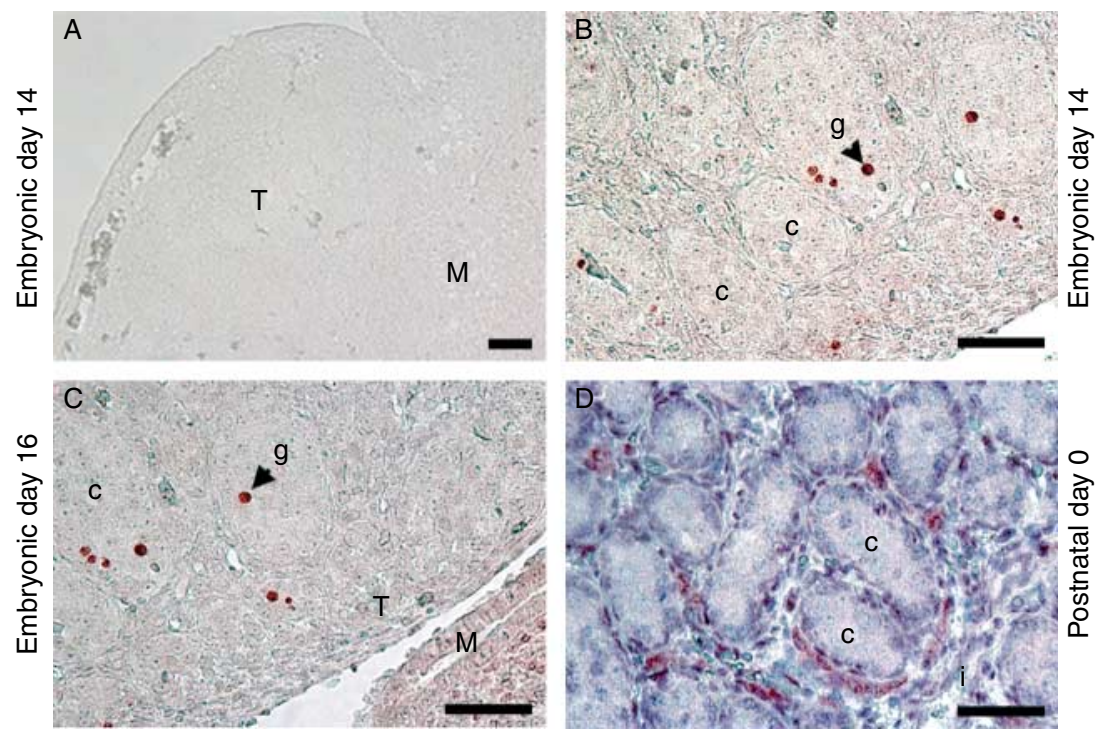

Figure 5 Immunohistochemistry for VEGFA antiangiogenic isoforms in E14 (B), E16 (C), and P0 (D) testes. D was counterstained with hemotoxylin. (A) E14 testis sections with no primary antibody served as negative controls. These data are the result of at least three different testes from each developmental time point. Scale bar represents $50 \mu \mathrm{m}$. T, testis; $M$, mesonephros; c, seminiferous cord; g, germ cell; i, interstitium. of germ cells between E14 and E18 in the developing rat testis (Levine et al. 2000). Therefore, increased expression of Vegfa_164 may be important in promoting increased blood flow and nutrients to the growing testis, or VEGFA_164 may have a more direct role in supporting the proliferation of Sertoli and/or germ cells.

Levels for Vegfa_165b mRNA were greatest between E13.5 and E16 in the testis. An increase in the expression of the antiangiogenic Vegfa_165b may prevent excessive activity of proangiogenic isoforms, thus, preventing hypervascularization or other detrimental effects on the developing testis. For example, treatment of E13 organ culture testes with recombinant VEGFA_120 and VEGFA_164 for 3 days in the current study resulted in increased vascular density and disruption of cord formation.

The Vegfa_120 mRNA isoform was more abundant in the testis at both E13 (just before seminiferous cord formation) and E14 (just after cord formation). Quantification of our western blots revealed that VEGFA_120/121B was more abundant than VEGFA_164/165B, VEGFA188/189B, and VEGFA_205/ 206B at E16-18. The VEGFA_120 isoform is believed to play a role in endothelial cell recruitment (Grunstein et al. 2000), and the migration of mesonephric endothelial cells and the formation of a distinct vascular pattern which occurs in the testis do not occur in the ovary. Instead, ovarian vascular development results from the proliferation and extension of pre-existing gonadal endothelial cells and vessels (Coveney et al. 2008). Taken together, this suggests that VEGFA_120 may not only be critical for mesonephric endothelial cell migration and the formation of a sex-specific vasculature in the testis but may also be involved with the development of seminiferous cords.
mRNA levels for Vegfa_165b tended to be greater in the ovary than in the testis at E13. Less expression of Vegfa_165b and thus, reduced inhibition of proangiogenic isoforms in the testis may help to promote endothelial cell migration and coelomic vessel formation. Additionally, in our western blotting experiments, VEGFA_165B was the most abundant antiangiogenic isoform in the testis from E18 to P3. This implies that the VEGFA_165B isoform is the predominant antiangiogenic isoform in the developing testis involved in the regulation of the angiogenic or possibly even nonangiogenic effects of the proangiogenic VEGFA isoforms.

Our QRT-PCR and western blotting experiments resulted in varied abundance patterns between the mRNA and protein for the different VEGFA isoforms. Even though the cause for these discrepancies is not clear, it may be the result of differences in posttranscriptional processing, and our laboratory is investigating this issue further.

Protein for antiangiogenic VEGFA isoforms was localized to the subsets of germ cells in E14-16 testes but little to no antiangiogenic VEGFA protein was present within seminiferous cords after birth. Other laboratories have demonstrated that treatment with proangiogenic VEGFA isoforms increases germ cell proliferation and survival in bovine testis explants (Caires et al. 2009). Therefore, the subset of germ cells expressing antiangiogenic VEGFA in embryonic testes may be apoptotic germ cells. The staining for antiangiogenic VEGFA isoforms in the embryonic testis was localized to the nucleus of the germ cells. Previous studies have localized VEGFA staining to the cytoplasm of rodent testicular cells (Nalbandian et al. 2003, Bott et al. 2006) and to what appears to be the nucleus of mitotically active bovine germ cells (Caires et al. 2009). 

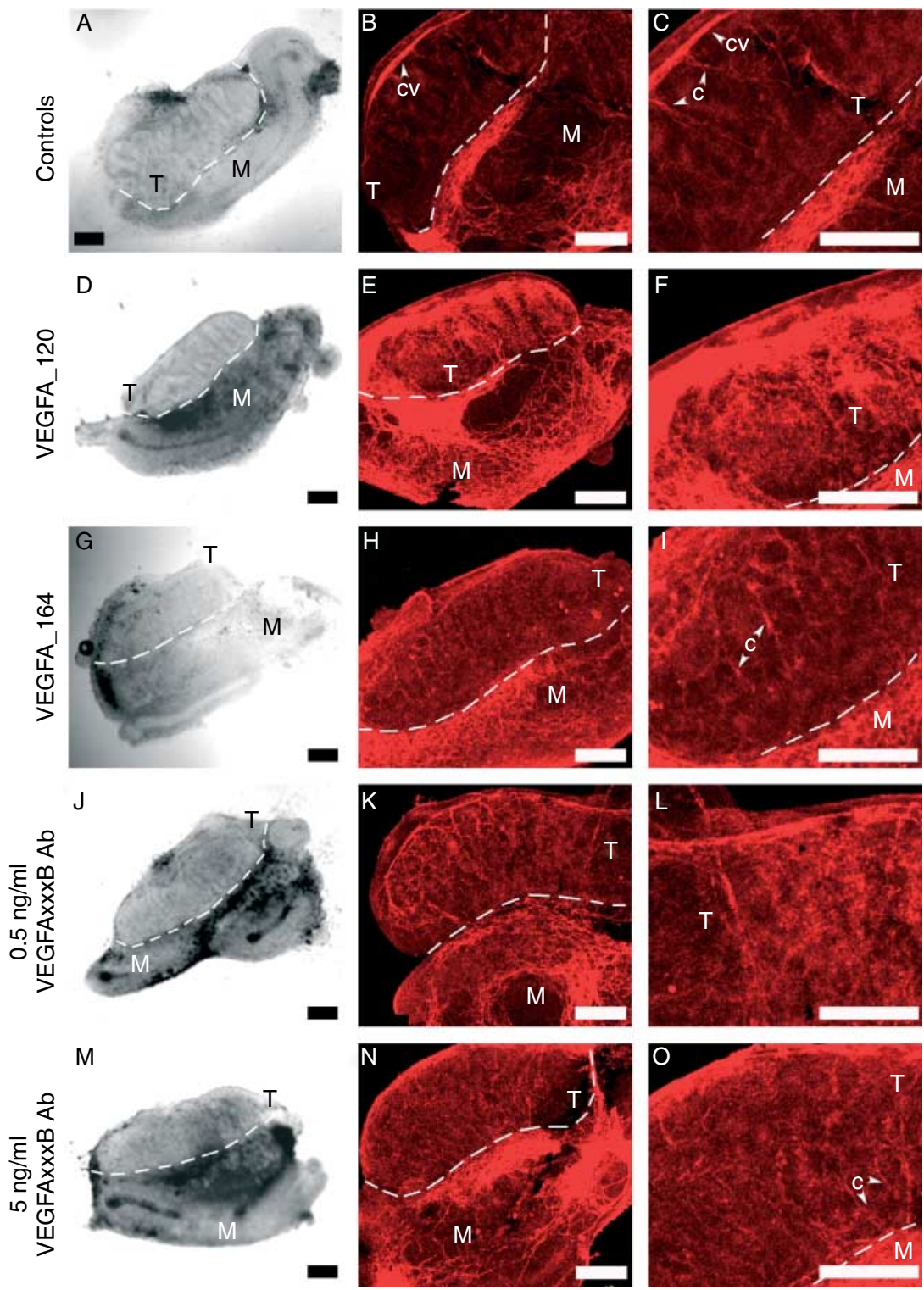

Figure 6 Embryonic day 13 testis organ cultures without treatment $(\mathrm{A}-\mathrm{C})$ or treatment with $50 \mathrm{ng} / \mathrm{ml}$ of recombinant VEGFA_120 (D-F), $50 \mathrm{ng} / \mathrm{ml}$ of recombinant VEGFA_164

(G-l), $0.5 \mathrm{ng} / \mathrm{ml}$ of VEGFAxxxB antibody (J-L), and $5 \mathrm{ng} / \mathrm{ml}$ of VEGFAxxxB antibody (M-O). (A, D, G, J, and M) Brightfield images. (B, C, E, F, $\mathrm{H}, \mathrm{I}, \mathrm{K}, \mathrm{L}, \mathrm{N}$, and $\mathrm{O}$ ) Confocal images of whole mount immunohistochemistry staining for PECAM1 (red indicates endothelial cell marker) to localize vasculature. Scale bar represents $200 \mu \mathrm{m}$. T, testis; M, mesonephros; c, cord.
The localization of antiangiogenic VEGFA to the nucleus of germ cells in this study is intriguing, and warrants further investigation. If antiangiogenic VEGFA isoforms are associated with apoptotic germ cells, cellular compromise or nuclear fragmentation may allow for the nuclear binding of these isoforms. Our lab has also shown that VEGFA is expressed in germ cell cytoplasm and Sertoli cells from E14 through P5, and that KDR is present in Sertoli and germ cells at P0 and P5 (Bott et al. 2006). Previous studies have shown that there is a decrease in germ cell proliferation between E14 and P0 followed by an increase in proliferation between $\mathrm{PO}$ and P5 (Levine et al. 2000). Taken together, these data suggest that interactions between pro- and antiangiogenic VEGFA isoforms may have in role in germ cell proliferation and/or maturation.
Organ culture testes treated with recombinant VEGFA_120 and VEGFA_164 developed intense vascularization between seminiferous cords, had thick coelomic vessels, and had greater vascular density compared with controls. Other studies have demonstrated that overexpression of VEGFA isoforms leads to infertility in mice. Transgenic mice that overexpress VEGFA_165 in the testis and epididymis showed complete infertility (Korpelainen et al. 1998), while mice that overexpress VEGFA_120 had reduced or complete infertility due to impaired spermiogenesis (Huminiecki et al. 2001). In the current study, the disruption of normal seminiferous cord formation may be due to the increased vascularization.

Organ culture testes treated with VEGFAxxxB antibody also developed intense vascularization and 


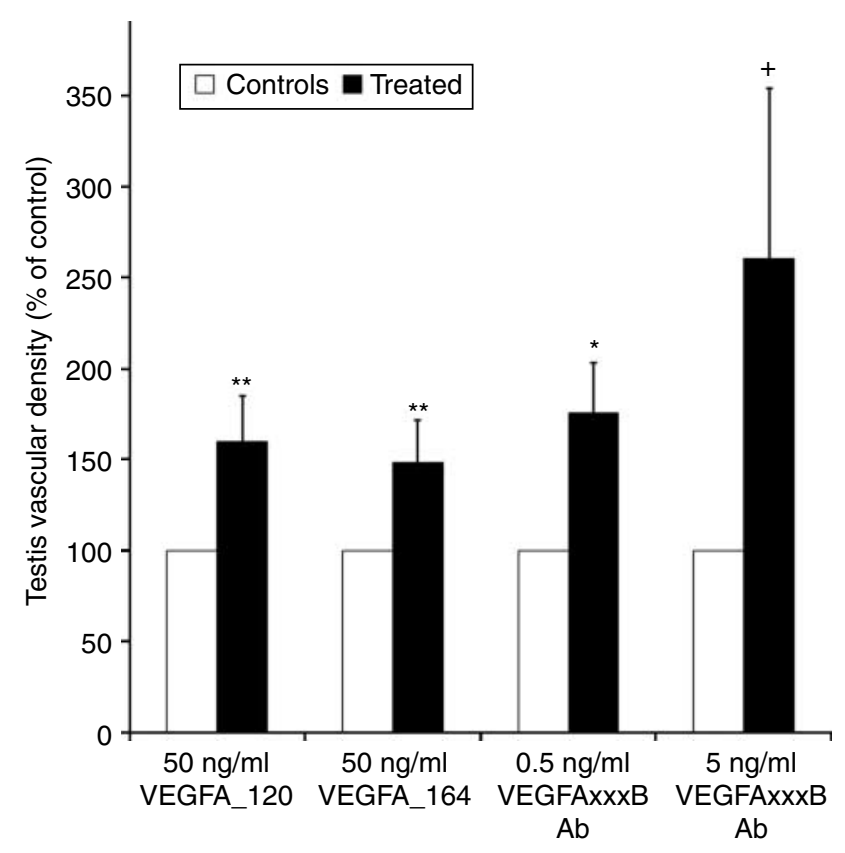

Figure 7 Effect of VEGFA_120, VEGFA_164, 0.5 ng/ml VEGFAxxxB antibody, and $5 \mathrm{ng} / \mathrm{ml}$ VEGFAxxxB antibody on vascular density in cultured testes expressed as a percentage of control organs. Data are the result of 21 (A (VEGFA_120 and VEGFA_164)), 24 (A (0.5 ng/ml VEGFAxxxB antibody)), and 22 (A (5 ng/ml VEGFAxxxB antibody)) testis pairs. The mean \pm s.E.M. stained areas for PECAM1 (vascular density) are presented for each organ culture treatment. Asterisks represent a statistically significant difference between treated and control groups $\left({ }^{*} P<0.008,{ }^{* *} P<0.05\right)$. The plus sign indicates a tendency toward different means $\left({ }^{+} P<0.09\right)$. Ab, antibody.

perturbed cord formation. The VEGFAxxxB antibody binds the antiangiogenic VEGFA ' $B$ ' isoforms, preventing them from blocking proangiogenic isoforms from binding to their receptors. Binding of antiangiogenic isoforms to VEGFAxxxB antibody should therefore allow the endogenous VEGFA proangiogenic isoforms to act at their receptors to increase endothelial cell migration and proliferation.

The results from the current study, along with those from previous studies (Bott et al. 2006), support a role for VEGFA in testis morphogenesis and vascular development. This study also demonstrated that antiangiogenic VEGFA isoforms are critical during early testis morphogenesis. mRNA for Vegfa_120 is more abundant in the testis, while Vegfa_164 and Vegfa_165b are more abundant in the ovary during the time when endothelial cells are migrating into the testis and testis-specific vasculature is developing. Furthermore, the expression of antiangiogenic VEGFA isoforms in germ cells in the embryonic testis suggests that the antiangiogenic isoforms may play a role in germ cell maturation. While organ culture testes treated with both proangiogenic isoforms and an antiangiogenic isoforms antibody resulted in hypervascularization and perturbed cord formation, treatment with the VEGFAxxxB antibody resulted in the greatest increase in vascular density. In conclusion, both pro- and antiangiogenic isoforms play a role in the development of rat testes, and differential expression of these isoforms may be critical for normal gonadal development.

\section{Materials and Methods \\ Animals}

Embryonic and postnatal ovaries were obtained from our own Sprague-Dawley rat colony at the University of NebraskaLincoln Animal Science Department, with founders purchased from Charles River (Wilmington, MA, USA). Testes were dissected from E13 to P3 rats in order to evaluate testes shortly before, during, and after seminiferous cord formation and testis-specific vascular development. To verify the sex of rat embryos utilized prior to morphological sex determination, Sry PCR was performed on genomic DNA according to previously reported methods (Anway et al. 2005). Embryonic age was calculated using plug date as E0. Postnatal age was determined using day of birth as P0. All animal procedures were approved by the University of Nebraska Animal Care and Use Committee.

\section{Vegfa isoforms RT-PCR and QRT-PCR}

Testes were dissected from rats at different ages, and mesonephroi were removed if present. Total RNA from these testes was isolated and converted to cDNA for subsequent RT-PCR according to standard protocols in our laboratory (McFee et al. 2009). The forward primer utilized for Vegfa antiangiogenic isoform conventional RT-PCR has been previously used for Vegfa proangiogenic (McFee et al. 2009) and antiangiogenic (Artac et al. 2009) isoform RT-PCR, and the reverse primer has been previously used for Vegfa antiangiogenic isoform RT-PCR (Artac et al. 2009). These primers were used with an annealing temperature of $54.5^{\circ} \mathrm{C}$ for 35 cycles to generate a 220-bp product for Vegfa_165b. The constitutively expressed gene, Gapdh, was used as a control for RNA isolation and amplification (Wesolowski et al. 2003), and previously reported methods (McFee et al. 2009) were utilized to produce a $460-\mathrm{bp}$ product. All PCR products were subcloned and confirmed using restriction digest analysis. PCR products were subcloned into pCRII (Invitrogen) using the TOPO TA Cloning kit (Invitrogen) and sequenced with primers for the T7 promoter region (data not shown). QRT-PCR primers and probes for rat Vegfa_120 (McFee et al. 2009), Vegfa_164 (McFee et al. 2009), and Vegfa_165b (Artac et al. 2009) have been used in prior experiments in our laboratory. Experimental and Gapdh PCRs were carried out in separate wells in triplicate. An arbitrary value of template was assigned to the highest standard and corresponding values to the subsequent dilutions. These relative values are plotted against the threshold value for each dilution to generate a standard curve. The relative amount for each experimental and Gapdh triplicate was assigned an arbitrary value based on the slope and $y$-intercept of the standard curve. The average of the experimental triplicate is divided by the average of the Gapdh 
triplicate, and the resulting normalized values are used for statistical analysis (McFee et al. 2009). RT-PCR and QRT-PCR were conducted on 3-5 different pools of tissue samples for each developmental time point.

\section{Protein extraction and western blotting for VEGFA isoforms}

Testes were collected from rats at different ages (if present, mesonephroi were removed), snap frozen in liquid nitrogen, and stored at $-80{ }^{\circ} \mathrm{C}$. Tissues were homogenized in a glass tissue grinder by mixing four organs with $60 \mu \mathrm{l}$ of homogenization buffer: $50 \mathrm{mM}$ Tris- $\mathrm{HCl}, \mathrm{pH} 7.4,150 \mathrm{mM} \mathrm{NaCl}, 10 \mathrm{mM}$ EDTA, $0.1 \%$ SDS, $100 \mathrm{mM}$ phenylmethanesulfonyl fluoride, 1:50 Protease Inhibitor cocktail (Sigma-Aldrich), 1.5\% Triton $\mathrm{X}-100$, and $2 \mu \mathrm{M}$ DL-dithiothreitol. The tissue and buffer mixture was centrifuged for $10 \mathrm{~min}$ at $4{ }^{\circ} \mathrm{C}$, and the supernatant was collected, aliquoted, and stored at $-80^{\circ} \mathrm{C}$. Protein samples $(28 \mu \mathrm{g}$ protein: proangiogenic, 15 or $50 \mu$ g protein: antiangiogenic) were mixed with $12.5-\mu$ l sample buffer (62.5 mM Tris- $-\mathrm{HCl}, \mathrm{pH} 6.8,20 \%$ glycerol, 2\% SDS, 5\% $\beta$-mercaptoethanol, trace Orange G) and enough Millipore water to bring total mixture volume to $40 \mu \mathrm{l}$. Samples were then incubated at $95{ }^{\circ} \mathrm{C}$ for $5 \mathrm{~min}$ and loaded onto 12 or $15 \%$ polyacrylamide separating gels for electrophoresis. We utilized the higher concentration gels for three of our antiangiogenic western blots to provide better separation of the VEGFA isoforms. Pre-stained protein standards (Bio-Rad) were utilized to verify transfer efficiency and to approximate protein molecular weight.

Proteins were electrotransferred onto polyvinylidene fluoride membranes (Immobilon transfer membranes; Millipore, Billerica, MA, USA) with a semi-dry blotting apparatus (Bio-Rad). Prior to transfer, the gel was soaked in transfer buffer (48 mM Tris- $\mathrm{HCl}, 39 \mathrm{mM}$ glycine, $0.0375 \%$ SDS, and $20 \%$ methanol) twice for $10 \mathrm{~min}$, the blotting paper (Bio-Rad) was soaked in transfer buffer for $5 \mathrm{~min}$, and the membrane was soaked in $20 \%$ methanol for 2-3 min. After transfer, the membrane was blocked with Odyssey blocking buffer (LI-COR Biosciences, Lincoln, NE, USA) for $2 \mathrm{~h}$ at room temperature, and then incubated overnight with primary antibody at $4{ }^{\circ} \mathrm{C}$ with gentle shaking. After incubation with the primary antibody, the membrane was washed three times for $10 \mathrm{~min}$ at room temperature in Tris-buffered saline with Tween (TBST) wash solution. The membrane was then incubated for $1 \mathrm{~h}$ with the secondary antibody at room temperature with gentle shaking. This and all subsequent steps were carried out without exposing the membrane to light. After incubation with the secondary antibody, the membrane received three additional 10-min washes in TBST at room temperature.

The VEGFA primary antibody was a goat polyclonal IgG raised against an epitope mapping at the $\mathrm{N}$-terminus of mouse VEGFA (catalog number: sc-1836; Santa Cruz Biotechnology, Santa Cruz, CA, USA). The VEGFA antiangiogenic isoforms primary antibody was a mouse monoclonal $\lg \mathrm{G}_{1}$ raised against a peptide corresponding to the 9-amino acid C-terminus of human VEGFA_165B (catalog number: ab14994; Abcam, Cambridge, MA, USA). This antibody has been characterized previously to bind VEGFA_165B (Woolard et al. 2004) and
VEGFA_121B (Rennel et al. 2009), and may also bind VEGFA_145B, VEGFA_183B, and VEGFA_189B (Perrin et al. 2005). Therefore, this antibody is collectively termed VEGFAxxxB but is sold commercially (Abcam and R\&D Systems, Minneapolis, MN, USA) as a VEGFA_165B antibody. The secondary antibodies utilized were Alexa Fluor 680 rabbit anti-goat IgG (Invitrogen) and IRDye 680 goat anti-mouse IgG (LI-COR Biosciences). The VEGFA primary antibody was diluted 1:100, the VEGFAxxxB antibody was diluted 1:1000, and the secondary antibodies were diluted 1:6000 in blocking buffer. Recombinant mouse VEGFA_164 and VEGFA_165B (R\&D Systems) were utilized as loading controls for VEGFA antibody and VEGFAxxxB antibody blots respectively.

Images of blots were acquired with the Odyssey infrared imaging system, and the Odyssey software program (LI-COR Biosciences) was utilized for quantitative analysis. For each individual blot, a standard curve was established from the densitometry measurement of the recombinant protein loading control, and the densitometry values of the VEGFA bands were quantified using this standard curve. Since different quantities of protein samples were utilized for the antiangiogenic western blots, densitometry values were proportionally adjusted by the amount of sample protein used to allow for standardized comparison of values. Quantification of each VEGFA isoform was performed on 3-6 blots from each developmental time point.

\section{Immunohistochemistry for VEGFA antiangiogenic isoforms}

Testes were histologically prepared, and immunohistochemistry was performed according to the standard protocols in our laboratory (McFee et al. 2009). The primary antibody was a VEGFAxxxB antibody (Abcam, the same antibody used for western blotting). As a negative control, serial sections were processed without primary antibody. The biotinylated goat antimouse secondary antibody was diluted 1:300. The secondary antibody was detected with aminoethyl carbazole chromagen substrate solution (Zymed Laboratories, South San Francisco, CA, USA). Immunohistochemistry was performed on at least three different sections of tissue from each age group.

\section{Organ cultures}

Testes with attached mesonephroi from E13 rats were cultured in accordance with previously reported organ culture methods (Bott et al. 2006). We cultured E13 gonads and mesonephroi from embryos with 15-18 tail somites since this stage of embryonic testis development immediately precedes mesonephric cell migration and seminiferous cord formation (Hacker et al. 1995, Uzumcu et al. 2002, Bott et al. 2008) and similar culture methods have demonstrated that control testes will develop cords and testis-specific vasculature with 3 days of culture (Bott et al. 2006). One testis from each animal was designated as a control, while its pair was treated with either $50 \mathrm{ng} / \mathrm{ml}$ of recombinant VEGFA_120 (R\&D Systems), $50 \mathrm{ng} / \mathrm{ml}$ of recombinant VEGFA_164 (R\&D Systems), $5 \mathrm{ng} / \mathrm{ml}$ of VEGFAxxxB antibody, or $50 \mathrm{ng} / \mathrm{ml}$ of VEGFAxxxB antibody (Abcam, the same antibody utilized for western blotting and 
immunohistochemistry). Doses of VEGFA_120, VEGFA_164, and VEGFAxxxB antibody (each diluted in PBS with $0.1 \%$ BSA) were added directly to the culture medium of the treated wells at the start of culture, and treatment was repeated daily. Similar doses of PBS with $0.1 \%$ BSA were added to the paired control wells for the VEGFA_120 and VEGFA_164 cultures, while nonspecific IgG in PBS with $0.1 \%$ BSA was added to the paired control wells for the VEGFAxxxB antibody cultures. Culture media were changed every other day. Only organ pairs in which control testes formed cords were used for analysis. All control testes in the VEGFA_164 (32 out of 32) and $0.5 \mathrm{ng} / \mathrm{ml}$ VEGFAxxxB antibody (25 out of 25) experiments formed cords, and all control testes but one in the VEGFA_120 (24 out of 25) and $5 \mathrm{ng} / \mathrm{ml}$ VEGFAxxxB antibody (21 out of 22) experiments formed cords by the end of culture.

\section{Imaging and area analysis of organ cultures}

After culture treatment, gonads were imaged to obtain individual testis area as previously described (McFee et al. 2009). The area for each testis was measured three times, and these three areas were averaged to obtain an accurate area measurement. The mean area for each control organ was set to $100 \%$, and the mean of each treated organ was calculated as a percentage of its paired control. A total of $17(5 \mathrm{ng} / \mathrm{ml}$ VEGFAxxxB antibody), 18 (0.5 ng/ml VEGFAxxxB antibody), and 20 (VEGFA_120 and VEGFA_164) testis pairs were imaged for area measurements.

\section{Whole mount immunohistochemistry of organ cultures and vascular density quantification}

After imaging, gonads were fixed in $4 \%$ paraformaldehyde for whole mount immunohistochemistry. Whole mount immunohistochemistry for PECAM1 utilized a mouse monoclonal $\operatorname{lgG}_{1}$ primary antibody raised against rat platelet endothelial cell adhesion molecule (BD Pharmingen, San Diego, CA, USA) and followed the same procedures used for our previously reported VEGFA antagonist (V1) treated ovary culture experiments (McFee et al. 2009). Confocal microscopy was performed, and the PECAM1 density was evaluated for control and treated organ culture gonads as previously described (McFee et al. 2009). Merged (all confocal z-section images merged together) red channel confocal images were used to analyze the PECAM1 density, and the vascular density or staining index was quantified using the National Institutes of Health Scion Image program (Scion Image, Frederick, MD, USA). Densitometry was performed on three, non-overlapping fields for each organ using a digital zoom at three times the original image that was acquired at $100 \times$ magnification. Within each field, the staining index was defined as the number of pixels exceeding an arbitrary grey scale value. The mean staining index for each testis was defined as the average of the staining indexes from all three fields. The mean density for each control testis was set to $100 \%$, and the mean of each treated testis was calculated as a percentage of its paired control. A total of 20 (VEGFA_120), 21 (5 ng/ml VEGFAxxxB antibody), $24(0.5 \mathrm{ng} / \mathrm{ml}$ VEGFAxxxB antibody), and 27 (VEGFA_164) testis pairs were analyzed for PECAM1 density.
In the embryonic gonad, PECAM1 is a marker for both vascular endothelial cells and primordial germ cells; however, localization of PECAM1 expression to germ cells diminishes after the morphological differentiation of male and female gonads (Wakayama et al. 2003). Visual analysis of the confocal images did not reveal any apparent differences between the PECAM1-positive germ cells in treated testes and those in their paired controls; however, there were obvious differences in vasculature across organ pairs. Therefore, any variation in PECAM1 density for treated organ compared with controls was considered to be primarily the result of changes in vascular density.

\section{Statistical analysis}

All data were analyzed by one-way ANOVA using JMP software (SAS Institute, Cary, NC, USA). Student's $t$-test was used to compare mean normalized QRT-PCR values between different developmental ages and to compare western blot quantification values between different VEGFA isoforms and across developmental ages. Student's $t$-test and a Dunnett test were used to compare area, thickness, and vascular density between control and treated organs. Differences in data were considered to be statistically significant at $P<0.05$ unless otherwise stated.

\section{Declaration of interest}

The authors declare that there is no conflict of interest that could be perceived as prejudicing the impartiality of the research reported.

\section{Funding}

This research was supported by NIH/NICHDRO1-HD051979 and a contribution of the University of Nebraska Agriculture Research Division (Lincoln, Nebraska).

\section{Acknowledgements}

We thank Drs Jess Miner and Brett White for critical reading of this manuscript; Dr Joe Zhou and his staff at the University of Nebraska Microscopy Core for their assistance; and Monika Burocziova, Jocelyn Wiarda, Robyn Longfellow Smith, Natalie Hart, and Shantille Kruse for technical assistance.

\section{References}

Anway MD, Cupp AS, Uzumcu M \& Skinner MK 2005 Epigenetic transgenerational actions of endocrine disruptors and male fertility. Science 308 1466-1469.

Artac RA, McFee RM, Longfellow Smith RA, Baltes-Breitwisch MM, Clopton DT \& Cupp AS 2009 Neutralization of vascular endothelial growth factor antiangiogenic isoforms is more effective than treatment with proangiogenic isoforms in stimulating vascular development and follicle progression in the perinatal rat ovary. Biology of Reproduction 81 978-988. 
Bates DO, Cui TG, Doughty JM, Winkler M, Sugiono M, Shields JD, Peat D, Gillatt D \& Harper SJ 2002 VEGF165b, an inhibitory splice variant of vascular endothelial growth factor, is down-regulated in renal cell carcinoma. Cancer Research 62 4123-4131.

Bott RC, McFee RM, Clopton DT, Toombs C \& Cupp AS 2006 Vascular endothelial growth factor and kinase domain region receptor are involved in both seminiferous cord formation and vascular development during testis morphogenesis in the rat. Biology of Reproduction $\mathbf{7 5}$ 56-67.

Bott RC, Clopton DT \& Cupp AS 2008 A proposed role for VEGF isoforms in sex-specific vasculature development in the gonad. Reproduction in Domestic Animals 43 310-316.

Brennan J, Karl J \& Capel B 2002 Divergent vascular mechanisms downstream of Sry establish the arterial system in the XY gonad. Developmental Biology 244 418-428.

Buehr M, Gu S \& McLaren A 1993 Mesonephric contribution to testis differentiation in the fetal mouse. Development 117 273-281.

Caires KC, de Avila J \& McLean DJ 2009 Vascular endothelial growth factor regulates germ cell survival during establishment of spermatogenesis in the bovine testis. Reproduction 138 667-677.

Capel B, Albrecht KH, Washburn LL \& Eicher EM 1999 Migration of mesonephric cells into the mammalian gonad depends on Sry. Mechanisms of Development 84 127-131.

Combes AN, Wilhelm D, Davidson T, Dejana E, Harley V, Sinclair A \& Koopman P 2009 Endothelial cell migration directs testis cord formation. Developmental Biology 326 112-120.

Cool J, Carmona FD, Szucsik JC \& Capel B 2008 Peritubular myoid cells are not the migrating population required for testis cord formation in the XY gonad. Sexual Development 2 128-133.

Coveney D, Cool J, Oliver T \& Capel B 2008 Four-dimensional analysis of vascularization during primary development of an organ, the gonad. PNAS 105 7212-7217.

Ferrara N, Gerber HP \& LeCouter J 2003 The biology of VEGF and its receptors. Nature Medicine 9 669-676.

Grunstein J, Masbad JJ, Hickey R, Giordano F \& Johnson RS 2000 Isoforms of vascular endothelial growth factor act in a coordinate fashion to recruit and expand tumor vasculature. Molecular and Cellular Biology 20 7282-7291.

Hacker A, Capel B, Goodfellow P \& Lovell-Badge R 1995 Expression of Sry, the mouse sex determining gene. Development 121 1603-1614.

Huminiecki L, Chan HY, Lui S, Poulsom R, Stamp G, Harris AL \& Bicknell R 2001 Vascular endothelial growth factor transgenic mice exhibit reduced male fertility and placental rejection. Molecular Human Reproduction 7 255-264.

Jost A, Magre S \& Agelopoulou R 1981 Early stages of testicular differentiation in the rat. Human Genetics $\mathbf{5 8} 59-63$.

Kidokoro T, Matoba S, Hiramatsu R, Fujisawa M, Kanai-Azuma M, Taya C, Kurohmaru M, Kawakami H, Hayashi Y, Kanai Y et al. 2004 Influence on spatiotemporal patterns of a male-specific Sox 9 activation by ectopic Sry expression during early phases of testis differentiation in mice. Developmental Biology 278 511-525.

Korpelainen EI, Karkkainen MJ, Tenhunen A, Lakso M, Rauvala $\mathbf{H}$, Vierula M, Parvinen M \& Alitalo K 1998 Overexpression of VEGF in testis and epididymis causes infertility in transgenic mice: evidence for nonendothelial targets for VEGF. Journal of Cell Biology 143 1705-1712.

Levine E, Cupp AS, Miyashiro L \& Skinner MK 2000 Role of transforming growth factor-alpha and the epidermal growth factor receptor in embryonic rat testis development. Biology of Reproduction 62 477-490.

Magre S \& Jost A 1980 The initial phases of testicular organogenesis in the rat. An electron microscopy study. Archives d'Anatomie Microscopique et de Morphologie Expérimentale 69 297-318.
Magre S \& Jost A 1991 Sertoli cells and testicular differentiation in the rat fetus. Journal of Electron Microscopy Technique 19 172-188.

Martineau J, Nordqvist K, Tilmann C, Lovell-Badge R \& Capel B 1997 Male-specific cell migration into the developing gonad. Current Biology 7 958-968.

McFee RM, Artac RA, McFee RM, Clopton DT, Longfellow Smith RA, Rozell TG \& Cupp AS 2009 Inhibition of vascular endothelial growth factor receptor signal transduction blocks follicle progression but does not necessarily disrupt vascular development in perinatal rat ovaries. Biology of Reproduction 81 966-977.

McLaren A 1991 Development of the mammalian gonad: the fate of the supporting cell lineage. BioEssays 13 151-156.

Merchant-Larios H, Moreno-Mendoza N \& Buehr M 1993 The role of the mesonephros in cell differentiation and morphogenesis of the mouse fetal testis. International Journal of Developmental Biology 37 407-415.

Nalbandian A, Dettin L, Dym M \& Ravindranath N 2003 Expression of vascular endothelial growth factor receptors during male germ cell differentiation in the mouse. Biology of Reproduction 69 985-994.

Park JE, Keller GA \& Ferrara N 1993 The vascular endothelial growth factor (VEGF) isoforms: differential deposition into the subepithelial extracellular matrix and bioactivity of extracellular matrix-bound VEGF. Molecular Biology of the Cell 4 1317-1326.

Perrin RM, Konopatskaya O, Qiu Y, Harper S, Bates DO \& Churchill AJ 2005 Diabetic retinopathy is associated with a switch in splicing from anti- to pro-angiogenic isoforms of vascular endothelial growth factor. Diabetologia 48 2422-2427.

Rennel ES, Varey AH, Churchill AJ, Wheatley ER, Stewart L, Mather S, Bates DO \& Harper SJ 2009 VEGF(121)b, a new member of the VEGF(xxx)b family of VEGF-A splice isoforms, inhibits neovascularisation and tumour growth in vivo. British Journal of Cancer 101 1183-1193.

Springer ML, Hortelano G, Bouley DM, Wong J, Kraft PE \& Blau HM 2000 Induction of angiogenesis by implantation of encapsulated primary myoblasts expressing vascular endothelial growth factor. Journal of Gene Medicine 2 279-288.

Tilmann C \& Capel B 1999 Mesonephric cell migration induces testis cord formation and Sertoli cell differentiation in the mammalian gonad. Development 126 2883-2890.

Uzumcu M, Westfall SD, Dirks KA \& Skinner MK 2002 Embryonic testis cord formation and mesonephric cell migration requires the phosphotidylinositol 3-kinase signaling pathway. Biology of Reproduction 67 1927-1935.

Wakayama T, Hamada K, Yamamoto M, Suda T \& Iseki S 2003 The expression of platelet endothelial cell adhesion molecule-1 in mouse primordial germ cells during their migration and early gonadal formation. Histochemistry and Cell Biology 119 355-362.

Wesolowski SR, Allan MF, Nielsen MK \& Pomp D 2003 Evaluation of hypothalamic gene expression in mice divergently selected for heat loss. Physiological Genomics 13 129-137.

Woolard J, Wang WY, Bevan HS, Qui Y, Morbidelli L, Pritchard-Jones RO, Cui TG, Sugiono M, Waine E, Perrin R et al. 2004 VEGF165b, an inhibitory vascular endothelial growth factor splice variant: mechanism of action, in vivo effect on angiogenesis and endogenous protein expression. Cancer Research 64 7822-7835.

Received 15 October 2009

First decision 23 November 2009

Revised manuscript received 6 May 2010

Accepted 10 May 2010 